Background Studies of Swedish track and field athletes have shown that there is a substantial risk of injury. None of these studies have investigated the potential role of how athletes perceive their injury risk, and how it may play a part in the occurrence of sport injuries.

Objective Explore how Swedish track and field athletes perceive their injury risk, and examine the potential correlation with prior injury experience.

Design A quantitative design, an exploratory study.

Setting Swedish junior elite track and field athletes.

Patients (or Participants) The convenience sample comprised 69 out of 97 Swedish junior elite track \& field athletes. The entry criteria included injury free when answering the questionnaire, active in track \& field, participated in at least one youth or junior national team between 2013 and 2017.

Interventions (or Assessment of Risk Factors) The participants had four weeks to fill out a two-part online questionnaire. The first part requested relevant personal information including previous injuries in the past 12 months; the second part consisted of 'The Perception of Risk of Injury Scale' (RISSc), but modified accordingly to the targeted sport.

Main Outcome Measurements RISSc scores were set as the dependent variable.

Results Non-significant results $(p=0,095)$ were found between gender and perceived injury risk. If an athlete reported more than one injury in the past 12 months, they perceived their re-injury risk $(p<0,025)$ to be higher. Significant results were found between perceived injury risk and the severity of the injury $(\mathrm{p}<0,006, \mathrm{r}=-0,32)$.

Conclusions Previous injury has a small correlation to perceived injury risk. It may be possible to reduce negative perceptions concerning re-injury in athletes with higher perceptions of injury risk. Awareness of re-injury should be increased among athletes with a history of severe injury. This study may serve as a springboard for additional research.

\section{RUNNING STYLE-DEPENDENT RISK FACTORS FOR PATELLOFEMORAL PAIN}

Julia Smakal, Nadja Jamrog, Bartosz Wojanowski. Privatpraxis Orthopädie, Dortmund, Germany

\subsection{6/bjsports-2021-IOC.138}

Background Patellofemoral-pain is a widespread problem among recreational-runners. Often it comes to training absenteeism because of pain at the proximal edge of the patella.

Objective To examine if there is a connection between the occurrence of patellofemoral pain and the running style.

Design Retrospective case-control-study.

Setting The running analyses all took place in the same $2 \mathrm{D}$ running lab. The selected analyses are of recreational and amateur runners, running at least $10 \mathrm{~km} /$ week, who were complaining of patellofemoral pain.

Patients (or Participants) There was a pool of 1013 runninganalyses in which the subjects complained of patellofemoral complaints (234 analyses), then all dates of recreational and competitive athletes (234 analyses) were first filtered out. This resulted in an analysis number of 113 analyses, which were included. All participants were examined by a sport medicine specialist and the diagnosis of patellofemoral pain syndrome were determined by X-ray and MRI imaging.
Interventions (or Assessment of Risk Factors) Primary contact with the ground, malposition of the legs, Achilles tendon angle, pelvic stability, knee inflection and lower leg swing were observed via a 2D running-analyse.

Main Outcome Measurements The 113 analyses were examined in terms of running technique and dynamic biomechanical misalignments. The evaluation was based on the 4-point model of Marquardt.

Results It was noteworthy that in 98.2\% ( $\mathrm{n}=111)$ of the examined subjects, the primary contact with the ground was via the heel. In $90.9 \%(n=101)$ of these subjects, there was also increased knee flexion in the medium support phase. This was also observed in the other two subjects without heel strike $(91.1 \%(\mathrm{n}=103)$ increased knee flexion).

Conclusions The primary contact of the heel and also an increased knee flexion in the medium support phase can increased the risk of patellofemoral-pain occurrence.

\section{A RISK FACTOR ANALYSIS FOR HEAD, NECK, AND FACE INJURIES BETWEEN US MEN AND WOMEN RUGBY-7S PLAYERS BY AGE-GROUPS}

${ }^{1,2}$ Christian Victoria, ${ }^{2}$ Danielle C Ompad, ${ }^{1,3,4}$ Shen-Ying Richard Ma, ${ }^{1,5}$ Meryle G Weinstein, 6,7,8,9,10 Robert C Cantu, ${ }^{11,12,13}$ Answorth A Allen, 1,3,14,15Victor Lopez. 'Rugby Research and Injury Prevention Group, Hospital for Special Surgery, New York, USA; ${ }^{2}$ New York University, College of Global Public Health, Urban Epidemiology Lab, New York, USA; ${ }^{3}$ Sports Performance Research Institute New Zealand, Auckland University of Technology, Auckland, New Zealand; ${ }^{4}$ University of Missouri, Missouri Orthopaedic Institute and Thompson Laboratory for Regenerative Orthopaedics, Columbia, USA; ${ }^{5}$ New York University, Steinhardt School of Culture, Education and Human Development, New York, USA; ${ }^{6}$ Center for the Study of Traumatic Encephalopathy, Boston University School of Medicine, Boston, USA; 'Department of Neurosurgery and Sports Medicine, Emerson Hospital, Concord, USA; ${ }^{8}$ Sports Neurology and Concussion Program, Brigham and Women's Hospital, Boston, USA; ${ }^{9}$ Concussion Legacy Foundation, Waltham, USA; ${ }^{10}$ World Rugby, Independent Concussion Group, Dublin, Ireland; ${ }^{11}$ Sports Medicine and Shoulder Service, Hospital for Special Surgery, New York, USA; ${ }^{12}$ National Basketball Association, New York Knickerbockers, New York, USA; ${ }^{13}$ USA Basketball, Colorado Springs, USA; ${ }^{14}$ USA Rugby Empire and New England Geographic Union Rugby Football Unions, New York/Boston, USA; ${ }^{15}$ Northeast Rugby Academy, USA Rugby Development Program and USOC-Community Olympic Development Program, New York/Boston, USA

\subsection{6/bjsports-2021-IOC.139}

Background Previous studies have highlighted the prevalence of head, neck, and face (HNF) injuries among male and female rugby-15s players; however, differences in risk-factors between sexes and age have not yet been examined in Rugby$7 \mathrm{~s}$.

Objective To identify risk factors of HNF injuries and sex risk differences among Rugby-7 players by age-groups.

Design Logistic regression analysis.

Setting USA Rugby tournaments/series and championships (U19 to Elite; 2010-2016).

Participants 1,307 $(68 \%=$ men, 31\%=women $)$ head neck and face injured U.S. Rugby-7s tournamnent players.

Assessment of Risk Factors A cross-sectional analysis was conducted using the RISERugby Injury Registry. Anthropometric data, injury mechanism, and other factors were tabulated by HNF injuries and sex. Logistic regression determined the relationship between sex and HNF injuries. A final multivariable model was used to calculate the probability of HNF injuries and differences between sex and age-groups.

Results From 2010-2016, 1,679 match injuries were seen $(68 \%=$ men, $32 \%=$ women $)$ injuries. A total of $474(28 \%)$ HNF injuries were documented. The most commonly 